Chirurg 2014 $\cdot 85: 350$

DOI 10.1007/s00104-014-2728-8

Online publiziert: 30. März 2014

(c) Springer-Verlag Berlin Heidelberg 2014

J. Reibetanz · C.-T. Germer

Klinik für Allgemein-, Viszeral-, Gefäß- und Kinderchirurgie, Universitätsklinikum Würzburg

\title{
Perioperative Kortisonsubstitution bei steroidtherapierten Patienten in der Kolorektalchirurgie
}

rativ auf eine Erhaltungsdosis bzw. komplett ausgeschlichen. Primärer Endpunkt war das Nichtauftreten einer orthostatischen Hypotonie am 1. postoperativen Tag (POD), sekundäre Endpunkte beinhalteten weitere perioperative Parameter (z. B. Fatigue, stationäre Aufenthaltsdauer, Komplikationen...).

\section{Hintergrund}

Zur Prävention einer perioperativen Nebenniereninsuffizienz stellt die hochdosierte perioperative Kortisonsubstitution als sog. „Stressdosis“ ein Standardvorgehen bei Patienten dar, die unter Dauersteroidmedikation stehen und sich einem (größeren) operativen Eingriff unterziehen müssen. Selbiges Vorgehen gilt strenggenommen auch für Patienten, die bis zu 12 Monate vor der Operation unter Steroidmedikation standen, da die Nebennierenfunktion bis zu ein Jahr supprimiert sein kann. Eine belastbare Evidenz für Notwendigkeit und Nutzen einer hochdosierten perioperativen Kortisonsubstitution in o. g. Fällen existiert jedoch nicht.

\section{Fragestellung und Methode}

Ziel dieser prospektiv-randomisierten, einfach verblindeten Studie war der $B e$ leg der Nichtunterlegenheit einer Lowdose-Steroidmedikation (LDS) im Vergleich zur High-dose-Medikation (HDS, „Stressdosis“) im Rahmen eines resezierenden Darmeingriffs bei Patienten mit chronisch entzündlichen Darmerkrankungen (CED). Patienten der HDS-Gruppe erhielten am Operationstag fraktioniert insgesamt $400 \mathrm{mg}$ Hydrokortison, LDS-Patienten (ebenfalls fraktioniert) lediglich eine Hydrokortisondosis äquivalent zu ihrer Dauermedikation. In beiden Gruppen wurde die Medikation postope-

\section{Ergebnisse}

Insgesamt 92 Patienten konnten zwischen September 2010 und März 2012 in die Studie eingeschlossen werden, von denen 49 in die LDS- und 43 in die HDS-Gruppe randomisiert wurden. In beiden Patientenkollektiven lag die präoperative Dosis an Prednisolonäquivalent oberhalb der Cushing-Schwelle (HDS: median $30 \mathrm{mg} /$ Tag bzw. LDS: median 20 mg/Tag, p=0,4) und auch die übrigen demographischen oder klinischen Charakteristika waren vergleichbar zwischen beiden Gruppen. Bezogen auf den primären Endpunkt der Studie - das Nichtauftreten einer orthostatischen Hypotonie am 1. POD - blieben 95\% der HDS-Gruppe und 96\% der LDS-Gruppe unauffällig. Ebenso zeigten sich in den übrigen perioperativen Parametern (Operationsdauer, Blutverlust, intraoperative Flüssigkeitssubstitution, Gesamtkomplikationsrate) keine Unterschiede zwischen den Kollektiven, es fand sich jedoch ein Trend zu mehr infektiösen Komplikationen in der HDS-Gruppe ( $16 \%$ vs. $4 \%, \mathrm{p}=0,2)$.

\section{Diskussion und Fazit des Reviewers}

Die vorliegende Arbeit zeigt auf hohem Evidenzniveau und mit ausreichender statistischer Power, dass mit Kortikoiden therapierte CED-Patienten hinsichtlich der Vermeidung postoperativer orthostatischer Hypotonien - als Ausdruck einer Nebennierenrindeninsuffizienz nicht von einer perioperativen Dosiserhöhung des Kortisons (als „,Stressdosis“) profitieren. In Anbetracht des ungünstigen Nebenwirkungsprofils einer höher dosierten Kortisontherapie sollten diese Patienten daher ausschließlich niedrigdosiert, also entsprechend ihrer Hausmedikation, substituiert werden. Zu einer vergleichbaren Schlussfolgerung kommt auch ein bereits 2008 publiziertes Review unter Einschluss von 315 Patienten aus 9 Studien: Eine routiniemäßige perioperative Dosiserhöhung der Kortisontherapie wird als nicht erforderlich erachtet, sofern die Hausmedikation weitergeführt wird [1]. Die aktuelle Studienlage stellt damit die Jahrzehnte lang geübte chirurgische und anästhesiologische Praxis der Applikation einer perioperativen "Stressdosis" bei Patienten unter laufender Kortisontherapie auf den Prüfstand!

\section{Korrespondenzadresse}

Prof. Dr. C.-T. Germer

Klinik für Allgemein-, Viszeral-, Gefäß- und Kinderchirurgie, Universitätsklinikum Würzburg, Oberdürrbacher Str. 6, 97080 Würzburg germer_c@ukw.de

Interessenkonflikt. J. Reibetanz und C.-T. Germer geben an, dass kein Interessenkonflikt besteht.

\section{Literatur}

1. Marik PE, Varon J (2008) Requirement of perioperative stress doses of corticosteroids: a systematic review of the literature. Arch Surg 143:1222-1226 